\title{
The application of nanotechnology in medicine: treatment and diagnostics
}

Nanomedicine 2014

Edinburgh, UK, 26-27 March 2014

The British Society for Nanomedicine (BSNM) [1], in collaboration with SELECTBIO, organized Nanomedicine 2014. BSNM is a registered charity created to allow open access for industry, academia, clinicians and the public to news and details of ongoing nanomedicine research. The Nanomedicine 2014 program provided insight across a number of emerging nanotechnologies spanning treatment to diagnostics. A key objective of the meeting was provision of opportunities to build collaborations and rationalize nanoenabled healthcare solutions.

\section{Application of organic nanocarriers in medicine}

Dr Davide Moscatelli (Politecnico di Milano) described work on biodegradable and biocompatible comb-like polymers (CLBs). Polyesters exhibit biocompatibility and biodegradability that makes them attractive for drug delivery. The presentation focused on biocompatible and biodegradable nanoparticles with tunable hydrophobicity and biodegradation kinetics, synthesized using novel CLB. The synthesis and characterization of polymers and nanoparticles based on polylactic acid (PLA), polycaprolactone (PCL) and polylactic-co-glycolic acid (PLGA) was detailed [2]. The proposed synthesis allows small and better controlled particle sizes than current synthetic methods such as nanoprecipitation which, in addition, involves the use of an organic solvent. Using the presented process it is relatively easy to tune nanoparticle features, and investigate the influence of parameters such as emulsifier type, feeding mode and macromonomer chain length. CLB obtained through the copolymerization of these novel macromonomers with PEGylated hydroxyethylmethacrylate monomers (HEMA-PEG) have been produced through surfactant-free polymerization [3].
Dr Christine Dufès (University of Strathclyde) discussed the possibility of using polymer nanomedicines to target cancer. The use of genes as medicines to treat cancer is limited by the lack of safe and efficacious delivery systems for selective delivery to tumors by intravenous administration, without secondary effects on healthy tissues. To address this, Dufès and colleagues demonstrated that conjugation of a polypropylenimine dendriplex to transferrin, whose receptors are overexpressed on numerous cancers, resulted in targeted gene expression after intravenous administration [4]. Moreover, the intravenous administration the delivery system-therapeutic DNA encoding TNF- $\alpha$ complex, led to rapid and sustained tumor regression over one month (90\% complete response, $10 \%$ partial response for A431 human epidermoid tumors) [4]. Tumor suppression for $60 \%$ of PC-3 and $50 \%$ of DU145 prostate tumors was also seen [5]. The treatment was well tolerated by the animals. The presentation proposed transferrin-bearing polypropylenimine as a promising delivery system for cancer therapy.

Professor Steve Rannard (University of Liverpool) gave a keynote address describing recent work on a new class of polymer-based nanoparticles, polydendrons [6]. Dendrimers
Andrew Owen $*, 1$, Christine Dufès ${ }^{2}$, Davide Moscatelli ${ }^{3}$, Eric Mayes ${ }^{4}$, Jonathan F Lovell ${ }^{5}$, Kattesh V Katti ${ }^{6}$, Konstantin Sokolov', Mariarosa Mazza ${ }^{8}$, Olivier Fontaine ${ }^{9}$, Steve Rannard ${ }^{10}$ \& Vicki Stone ${ }^{11}$ 'Department of Molecular \& Clinical Pharmacology, University of Liverpool, Liverpool L69 3GF, UK

${ }^{2}$ Strathclyde Institute of Pharmacy \& Biomedical Sciences, University of Strathclyde, Glasgow G4 ORE, UK ${ }^{3}$ Department of Chemistry, Materials \& Chemical Engineering, Politecnico di Milano, Milano, Italy

${ }^{4}$ Endomagnetics Ltd, Cambridge, CB4 OWG, UK

${ }^{5}$ Department of Biomedical Engineering \& Department of Chemical \& Biological Engineering, University at Buffalo, State University of New York, Buffalo, New York, NY 14260, USA ${ }^{6}$ Institute of Green Nanotechnology \& Cancer Nanotechnology Platform, Department of Radiology, University of Missouri, Columbia, MO, USA ${ }^{7}$ Department of Imaging Physics, University of Texas M.D. Anderson Cancer Center, Houston, TX 77030, USA ${ }^{8}$ Nanomedicine Lab, Centre for Tissue Injury \& Repair, Institute of Inflammation \& Repair, Faculty of Medical \& Human Sciences, University of Manchester, Manchester M13 9PT, UK

${ }^{9}$ Nanobiotix S.A, 60 rue de Wattignies, 75012 Paris, France

${ }^{10}$ Department of Chemistry, University of Liverpool, Liverpool, UK

${ }^{11 S}$ School of Life Sciences, Heriot-Watt University, Edinburgh, UK *Author for correspondence: aowen@liv.ac.uk

\section{Future : Medicine part of}


are highly branched macromolecules that have a distinct interior and exterior and are highly functional. One barrier to wide application of dendrimers is their expensive synthesis. Conversely, hyperbranched polydendrons are rapid to synthesize and maintain a number of the benefits of dendrimers. Recent preliminary data showing that careful design of the materials can tailor permeation through gut epithelium models and accumulate preferentially in macrophages were discussed. These results suggest potential for oral administration and the accumulation of encapsulated drugs within macrophages - an important site for some diseases like HIV and TB.

Dr Jonathan F Lovell (University at Buffalo) summarized recent developments of porphyrin-based nanovesicles. 'Porphysome' nanovesicles, formed from a porphyrin-lipid bilayer, are organic nanoparticles with inherent optical activity [7]. They have numerous uses for multimodal imaging and therapy. Porphyrinphospholipid (PoP) liposomes have lower percentages of porphyrin-lipid in the bilayer, and can be stably loaded with drugs or other cargo [8]. Upon exposure to a red laser, the liposomes pop open and release their contents.

Dr Mariarosa Mazza (University of Manchester) presented recent data on nanoparticle-mediated siRNA gene silencing against brain disorders. Peptide nanofibers (PNFs) may find applications as biomaterials and have been investigated for neuronal regeneration and brain delivery [9]. Positively charged amphiphilic peptides able to self-assemble into PNFs have been engineered and shown to be internalized by primary neurons and removed or degraded in the brain [10]. Due to their biocompatibility, biodegradability and chemical versatility, PNFs can be designed as tools for gene therapy. These fiber-shaped constructs can be exploited for development of effective siRNA therapeutics as molecular transporters for reversible control of gene expression on targets with pathophysiological relevance.

\section{Application of inorganic nanoparticles in medicine}

Professor Kattesh V Katti gave a keynote lecture on green nanotechnology in cancer therapy. The creation of biocompatible radioactive gold nanoparticles is possible using epigallocatechin gallate (EGCG), found in abundance in tea, as a reducing agent. This development is unique because the transformation of gold salt to the corresponding gold nanoparticles is achieved by simple mixing of EGCG (or tea leaves) with the gold precursor. The creation of therapeutic $\beta$-emitting $\mathrm{Au}-$ 198-radioisotope-based nanoparticles, encapsulated with EGCG, was also discussed. The laminin receptor specificity of EGCG (and the therapeutic EGCG-198AuNPs) allows resultant nanomedicines with hydrody- namic sizes between 50 and $65 \mathrm{~nm}$ to penetrate laminin receptor-expressing tumor vasculature. Development of therapeutic EGCG-198-AuNPs for treating solid prostate tumors was demonstrated in vivo [11]. The overall implications of green nanotechnology was summarized in the context of functionalized radioactive gold nanoparticles in oncology.

Dr Konstantin Sokolov (University of Texas M.D. Anderson Cancer Center) discussed recent work on a magneto-plasmonic nanoparticle platform for capture, separation and enumeration of rare cells [12]. Detection of disseminated tumor cells or tumor biomarkers in human fluids such as blood, urine, and saliva can provide an accessible tool for cancer detection and therapy monitoring. However, the challenge of detecting circulating tumor cells (CTCs) is their rare occurrence, estimated from one to several CTCs among millions of leukocytes and billions of erythrocytes. Dr Sokolov and co-workers have addressed this by developing nanoparticle probes with multiple functionalities. Molecular targeted magneto-plasmonic nanoclusters which exhibit strong red-NIR absorbance and superparamagnetic properties were synthesized. Data for simultaneous magnetic capture and photoacoustic detection of cancer cells in whole blood with greater than $90 \%$ capturing efficiency, with no laborious processing steps that are commonly used in other CTC assays was discussed. It was proposed that this platform provides a foundation for development of low cost simple and nearly realtime assays for capture, separation and enumeration of rare cells.

Dr Eric Mayes (Endomagnetics Ltd) discussed the application of magnetic nanoparticles in surgical oncology. The development of Endomagnetics' nanoparticlebased system to increase access to the standard of care in cancer staging was specifically elaborated and how the system provides an alternative approach, avoiding the requirement of radioisotopes for sentinel lymph node biopsy, improving workflow and costs while increasing access for patients [13].

\section{Pharmacokinetic benefits from solid drug nanoparticles}

An overview of the applications of solid drug nanoparticle (SDN) formulations was provided by Professor Andrew Owen (University of Liverpool). The talk focused on 'nanoparticle engineering' to form dispersions where each submicron particle consists of the drug. Nanomilling has been the most successful nanoparticleengineering technology commercially, and relies on the formation of SDNs with application for improving oral bioavailability (e.g., dalfampridine), overcoming food effects (e.g., megesterol acetate), modified delivery profile (e.g., ritalin) and sustained-release intramuscular 
depot formulations (e.g., paliperidone). Recent work on antiretroviral SDNs produced by a new efficient, scalable and versatile methodology was also presented. The approach has produced oral formulations of efavirenz with preclinical data indicating a dose reduction may be possible while maintaining plasma exposure [14]. The technology is being commercialized through a University of Liverpool start-up company called Tandem Nano Ltd [15].

\section{Overview of nanomaterial safety}

Professor Vicki Stone (Herriot Watt University) discussed in vitro and in vivo models to assess local and systemic responses to nanomaterial (NM) exposure [16]. The diversified use of NMs, including nanomedicine and diagnostics, imply that exposure can occur via several different routes for which reliable alternative models to animal testing are required. However, in vitro models are often questioned for their relevance [17]. Side-byside comparisons of a hepatocyte cell line (C3A) with rats exposed intravenously with $\mathrm{NMs}\left(\mathrm{TiO}_{2}, \mathrm{Au}\right.$ and $\mathrm{Ag}$ ) suggested that the hepatocyte model was effective at predicting liver responses in terms of oxidative stress and proinflammatory mediator production. In contrast, simple in vitro models were not accurate in predicting liver, intestine or aorta responses following gavage, or liver responses following intratracheal instillation. The presented data suggest that the gut and lungs may change particle properties, or that the particles stimulate gut to activate humoral/neural communication pathways. Adaptation of the method of dispersal to better reflect the route of entry (e.g., dispersion in lung lining fluid before serum) could help improve in vitro models and this was also discussed.

\section{Other presentations}

A number of other noteworthy presentations were presented at the meeting. Dr Marianne Ashford (AstraZeneca) provided an eloquent overview about context and challenges of therapeutic index in the pharmaceutical industry. The presentation discussed the importance of therapeutic index improvement for oncology and how nanoparticles can improve therapeutic index, with various case histories to underpin sentient points. Dr Delphine Felder-Flesch (Institut de Physique et Chimie des Materiaux de Strasbourg) provided an overview on some recent work on dendrimer nanomedicines, specifically discussing studies of the biodistribution and elimination of dendritic radiopharmaceuticals and dendronized iron oxides. Finally, Dr Rosana Simon-Vazquez (University of Vigo) discussed work with fluorescence and Fourier transform infrared spectroscopy spectroscopy illustrating how metal oxide nanoparticles induce conformational changes in some human plasma protein fractions.

\section{European Technology Platform on Nanomedicine \& NANOMED2020}

Olivier Fontaine (Nanobiotix) outlined the mission of European Technology Platform on Nanomedicine (ETPN) and the key outcomes of NANOMED2020 European project. The ETPN was launched in 2005 to define strategic research priorities, is led by industry and officially recognized by the European Commission as the official Nanomedicine representative. As the ideal entity for cross-fertilization and collaboration between stakeholders, the ETPN is striving for a sustainable, competitive and profitable nanomedicine sector in Europe. In June 2013, the ETPN published under NANOMED2020 a white paper that defines the needs and recommendations for successful translation of research into innovative products [18]. The pivotal feature of the Nanomedicine Translation Hub was also discussed, which is comprised of several subinitiatives:

- A Translational Advisory Board to provide strategic advice and support to projects;

\section{- A European Nano-Characterisation Laboratory;}

- The creation of good manufacturing practice pilot lines for the production of NM batches for clinical trials.

The Nanomedicine Map [19], the Nanomedicine Awards [20], the Nano World Cancer Day [21] and a short animated Nanomedicine Movie [22] are other notable outcomes of NANOMED2020 that were discussed at the meeting.

\section{Financial \& competing interests disclosure}

A Owen is co-inventor of patents relating to nanomedicines, is co-founder of a recent start-up company (Tandem Nano Ltd), has received research funding from Merck, Pfizer, AstraZeneca and Janssen, and consultancy from Merck and Norgine. M Mazza is co-inventor of patents relating to nanomedicines. $K$ Sokolov is co-inventor of patents relating to nanomedicines and a co-founder of a recent start-up company (NanoHybrids). S Rannard is co-inventor of patents relating to nanomedicines, is co-founder of a recent start-up company (Tandem Nano Ltd), has received funding from IOTA NanoSolutions Ltd and Unilever, and consultancy from Bayer Cropscience. E Mayes is Chief Executive Officer of Endomagnetics Ltd. V Stone has received research funding from GlaxoSmithKline and from Unilever as well as consultancy from GlaxoSmithKline. The authors have no other relevant affiliations or financial involvement with any organization or entity with a financial interest in or financial conflict with the subject matter or materials discussed in the manuscript apart from those disclosed.

No writing assistance was utilized in the production of this manuscript. 


\section{References}

1 British Society for Nanomedicine. www.BritishSocietyNanomedicine.org

2 Ferrari R, Yu YC, Morbidelli M, Hutchinson RA, Moscatelli D. epsilon-caprolactone-based macromonomers suitable for biodegradable nanoparticles synthesis through free radical polymerization. Macromolecules 44, 9205-9212 (2011).

3 Ferrari R, Colombo C, Casali C et al. Synthesis of surfactant free PCL-PEG brushed nanoparticles with tunable degradation kinetics. Int. J. Pharm. 453, 551-559 (2013).

4 Koppu S, Oh YJ, Edrada-Ebel R et al. Tumor regression after systemic administration of a novel tumor-targeted gene delivery system carrying a therapeutic plasmid DNA. J. Control. Release 143, 215-221 (2010).

5 Al Robaian M, Chiam KY, Blatchford DR, Dufès C. Therapeutic efficacy of intravenously administered transferrin-conjugated dendriplexes on prostate carcinomas. Nanomedicine (Lond.) 9(4), 421-434 (2014).

6 Hatton FL, Chambon P, McDonald TO, Owen A, Rannard SP. Hyperbranched polydendrons: a new controlled macromolecular architecture with self-assembly in water and organic solvents. Chem. Sci. 5, 1844-1853 (2014).

7 Lovell JF, Jin CS, Huynh E et al. Porphysome nanovesicles generated by porphyrin bilayers for use as multimodal biophotonic contrast agents. Nat. Mater. 10 324-332 (2011).

8 Carter KA, Shao S, Hoopes MI et al. Porphyrin-phospholipid liposomes permeabilized by near-infrared light. Nat. Commun. 5, 3546 (2014).

9 Mazza M, Notman R, Anwar J et al. Nanofiber-based delivery of therapeutic peptides to the brain. ACS Nano 7 , 1016-1026 (2013).

10 Mazza M, Patel A, Pons R, Bussy C, Kostarelos K. Peptide nanofibres as molecular transporters: from self-assembly to in vivo degradation. Faraday Discuss. 166, 181-194 (2013).
11 Shukla R, Chanda N, Zambre A et al. Laminin receptor specific therapeutic gold nanoparticles $\left({ }^{198} \mathrm{AuNP}\right.$-EGCg) show efficacy in treating prostate cancer. Proc. Natl Acad. Sci. USA 109, 12426-12431 (2012).

12 Wu CH, Huang YY, Chen P et al. Versatile immunomagnetic nanocarrier platform for capturing cancer cells. ACS Nano 7, 8816-8823 (2013).

13 Thill M, Kurylcio A, Welter R et al. The central-European SentiMag study: sentinel lymph node biopsy with superparamagnetic iron oxide (SPIO) vs. radioisotope. Breast 23, 175-179 (2014).

14 McDonald TO, Giardiello M, Martin P et al. Antiretroviral solid drug nanoparticles with enhanced oral bioavailability: production, characterization, and in vitro-in vivo correlation. Adv. Healthcare Mater. 3, 400-411 (2014).

15 Tandem Nano Limited. www.tandemnanano.com

16 Johnston H, Pojana G, Zuin S et al. Engineered nanomaterial risk. Lessons learnt from completed nanotoxicology studies: potential solutions to current and future challenges. Crit. Rev. Toxicol. 43, 1-20 (2013).

17 Stone V, Johnston H, Schins RP. Development of in vitro systems for nanotoxicology: methodological considerations. Crit. Rev. Tox. 39, 613-626 (2009).

18 Contribution of Nanomedicine to Horizon 2020. www.etp-nanomedicine.eu/etpn-white-paper-2013

19 European Nanomedicine Map. www.etp-nanomedicine.eu/public/european-nanomedicinemap

20 Nanomedicine Award 2013. http://nanomedicine-award.com/

21 Welcome to the NANO WORLD CANCER DAY 2014 ! www.nwcd.eu

22 About Nanomedicine. www.etp-nanomedicine.eu/public/about-nanomedicine 